\title{
A VARIABLE SPLITTING AUGMENTED LAGRANGIAN APPROACH TO LINEAR SPECTRAL UNMIXING
}

\author{
José M. Bioucas-Dias \\ Instituto de Telecomunicações, \\ Instituto Superior Técnico, Technical University of Lisbon, \\ Lisboa, Portugal
}

\begin{abstract}
This paper presents a new linear hyperspectral unmixing method of the minimum volume class, termed simplex identification via split augmented Lagrangian (SISAL). Following Craig's seminal ideas, hyperspectral linear unmixing amounts to finding the minimum volume simplex containing the hyperspectral vectors. This is a nonconvex optimization problem with convex constraints. In the proposed approach, the positivity constraints, forcing the spectral vectors to belong to the convex hull of the endmember signatures, are replaced by soft constraints. The obtained problem is solved by a sequence of augmented Lagrangian optimizations. The resulting algorithm is very fast and able so solve problems far beyond the reach of the current state-of-the art algorithms. The effectiveness of SISAL is illustrated with simulated data.
\end{abstract}

Index Terms - Hyperspectral unmixing, Minimum volume simplex, Variable Splitting augmented Lagrangian, nonsmooth optimization.

\section{INTRODUCTION}

Hyperspectral unmixing is a source separation problem [1]. Compared with the canonical source separation scenario, the sources in hyperspectral unmixing (i.e., the materials present in the scene) are statistically dependent and combine in a linear or nonlinear fashion. These characteristics, together with the high dimensionality of hyperspectral vectors, place the unmixing of hyperspectral mixtures beyond the reach of most source separation algorithms, thus fostering active research in the field [2].

Given a set of mixed hyperspectral vectors, linear mixture analysis, or linear unmixing, aims at estimating the number of reference materials, also called endmembers, their spectral signatures, and their abundance fractions [1, 2, 3, 4, 5, 6]. The approaches to hyperspectral linear unmixing can be classified as either statistical or geometrical. The former address spectral unmixing as an inference problem, often formulated under the Bayesian framework, whereas the latter exploit the fact that the spectral vectors, under the linear mixing model, are in a simplex whose vertices represent the sought endmembers.

\subsection{Statistical approach to spectral unmixing}

Modeling the abundance fractions (sources) statistical dependence in hyperspectral unmixing is a central issue in the statistical framework. In [7], the abundance fractions are modeled as mixtures of Dirichlet densities. The resulting algorithm, termed DECA (dependent component analysis), implements an expectation maximization iterative scheme for the inference of the endmember signatures (mixing matrix) and the density parameters of the abundance fractions.

The inference engine in the Bayesian framework is the posterior density of the entities to be estimated, given the observations.

This work was supported by the European Commission Marie Curie training grant MEST-CT-2005-021175. Email:\{bioucas\}@1x.it.pt
According to Bayes' law, the posterior includes two factors: the observation density, which may account for additive noise, and a prior, which may impose constraints on the endmember matrix (e.g., nonnegativity of its elements) and on the abundance fractions (e.g., to be in the probability simplex) and model spectral variability. Works [8. 9] are representative of this line of attack.

\subsection{Geometrical approach to spectral unmixing}

The geometrical approach exploits the fact that, under the linear mixing model, hyperspectral vectors belong to a simplex set whose vertices correspond to the endmembers. Therefore, finding the endmembers is equivalent to identifying the vertices of the referred to simplex.

If there exists at least one pure pixel per endmember (i.e., containing just one material), then unmixing amounts to finding the spectral vectors in the data set corresponding to the vertices of the data simplex. Some popular algorithms taking this assumption are the the N-FINDR [10], the the pixel purity index (PPI) [11], the automated morphological endmember extraction (AMEE) [12], the vertex component analysis (VCA) [4], and the simplex growing algorithm (SGA) [13].

If the pure pixel assumption is not fulfilled, what is a more realistic scenario, the unmixing process is a rather challenging task, since the endmembers, or at least some of them, are not in the data set. A possible line of attack, in the vein of the seminal ideas introduced in [6], is to fit a simplex of minimum volume to the data set. Relevant works exploiting this direction are the minimum volume enclosing simplex (MVES) [14], the minimum volume simplex analysys (MVSA) [15], and the nonnegative matrix factorization minimum volume transform (NMF-MVT) [16]. MVES and MVSA, although implemented in rather different ways, yield state-of-the-art results. Their major shortcoming is the time they take for more than, say, 10 endmembers and more than 5000 spectral vectors.

\subsection{Proposed approach}

We introduce the simplex identification via split augmented Lagrangian (SISAL) algorithm for unsupervised hyperspectral linear unmixing. SISAL belongs to the minimum volume class, and thus is able to unmix hyperspectral data sets in which the pure pixel assumption is violated.

In SISAL, the positivity hard constraints are replaced by hinge type soft constraints, whose strength is controlled by a regularization parameter. This replacement has three advantages: 1) robustness to outliers and noise; 2) robustness to poor initialization; 3) opens the door to dealing with large problems. Furthermore, for large values of the regularization parameter, the hard constraint formulation is recovered.

To tackle the hard nonconvex optimization problem we have in hands, we solve a sequence of nonsmooth convex subproblems, using variable splitting to obtain a constraint formulation, and then applying an augmented Lagrangian technique [17, 18]. This sub-problems implement an alternate minimization scheme, with very simple and fast steps. 
The paper is organized as follows. Section 2 formulates the problem. Section 3 proposes a sequence of nonsmooth subproblems, Section 4 solves the nonsmooth problems via a variable splitting augmented Lagrangian scheme, Section 5 presents results, and Section 6 ends the paper by presenting a few concluding remarks.

\section{PROBLEM FORMULATION}

Let us assume that in a given scene there are $p$ materials, termed endmembers, with spectral signatures $\mathbf{m}_{i} \in \mathbb{R}^{l}$, for $i=1 \ldots, p$, where $l \geq p$ denotes the number of spectral bands. Under the linear mixing model, a given hyperspectral observed vector is a linear combination of the endmember spectral signatures, where the weights represent the fractions that each material occupies in the pixel. Therefore, the observed spectral vectors are in the convex hull of endmember spectral signatures.

To fix notation, let $\mathbf{Y} \equiv\left[\mathbf{y}_{1}, \ldots, \mathbf{y}_{n}\right] \in \mathbb{R}^{l \times n}$ denote a matrix holding the observed spectral vectors $\mathbf{y}_{i} \in \mathbb{R}^{l}$ and $\mathbf{S} \equiv$ $\left[\mathbf{s}_{1}, \ldots, \mathbf{s}_{n}\right] \in \mathbb{R}^{p \times n}$ a matrix holding the respective fractions; i.e., $\mathbf{y}_{i}=\mathbf{M} \boldsymbol{s}_{i}$, for $i=1, \ldots, n$, where $\mathbf{M} \equiv\left[\mathbf{m}_{1}, \ldots, \mathbf{m}_{p}\right] \in \mathbb{R}^{p \times p}$ is the mixing matrix containing the endmembers, and $s_{i}$ is a vector denoting the fractions, often termed fractional abundances. Since the components of $\mathbf{s}_{i}$ are nonnegative and sum one (they are fractions), then the fractional abundance vectors belong to the standard $p$-simplex set $\mathcal{S}_{p}=\left\{\mathbf{s} \in \mathbb{R}^{p}: \mathbf{s} \succeq \mathbf{0}, \mathbf{1}_{p}^{T} \mathbf{s}=1\right\}$. Therefore,

$$
\mathbf{Y}=\mathbf{M S}, \quad \mathbf{S} \in \mathcal{S}_{p}^{n}
$$

Assuming that the endmember spectral signatures $\mathbf{m}_{i}$, for $i=$ $1, \ldots, p$, are linearly independent, then the set $\left\{\mathbf{y} \in \mathbb{R}^{l}: \mathbf{y}=\right.$ $\left.\mathbf{M s}, \mathbf{s} \in \mathcal{S}_{p}\right\}$ is a $(p-1)$-dimensional simplex, and estimating $\mathbf{M}$ amounts to infer its vertices. Figure 1 illustrates this perspective.

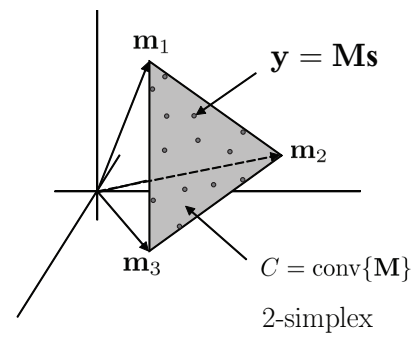

Fig. 1. Illustration of the 2-simplex set generated the columns of $\mathbf{M}$.

In this work, we assume that the number of endmembers and signal subspace is known before hand (see, e.g., [19]) and that the observed vectors $\mathbf{y}_{i}, i=1, \ldots, n$, represent coordinates with respect to a $p$-dimensional basis of the signal subspace, i.e., $l=p$.

Given $\mathbf{Y}$, and inspired by the seminal work [6], we infer matrices $\mathbf{M}$ and $\mathbf{S}$ by fitting a minimum volume simplex to the data subject to the constraints $\mathbf{S} \succeq 0$ and $\mathbf{1}_{p}^{T} \mathbf{S}=\mathbf{1}_{n}$. Since the volume defined by the columns of $\mathbf{M}$ is proportional to $|\operatorname{det}(\mathbf{M})|$ (note that we are assuming that $\mathbf{M}$ is square), then

$$
\begin{aligned}
\mathbf{M}^{*}= & \arg \min _{\mathbf{M}}|\operatorname{det}(\mathbf{M})| \\
\text { s.t. : } & \mathbf{Q Y} \succeq \mathbf{0}, \quad 1_{p}^{T} \mathbf{Q Y}=\mathbf{1}_{n}^{T},
\end{aligned}
$$

where $\mathbf{Q} \equiv \mathbf{M}^{-1}$. Since $\operatorname{det}(\mathbf{Q})=1 / \operatorname{det}(\mathbf{M})$, we can replace the problem (2) with

$$
\begin{aligned}
\mathbf{Q}^{*}= & \arg \min _{\mathbf{Q}}-\log |\operatorname{det}(\mathbf{Q})| \\
\text { s.t.: } & \mathbf{Q Y} \succeq \mathbf{0}, \quad \mathbf{1}_{p}^{T} \mathbf{Q Y}=\mathbf{1}_{n}^{T} .
\end{aligned}
$$

The constraints in (3) define a convex set. If matrix $\mathbf{Q}$ is symmetric and positive-definite, the problem (3) is convex. However, in

\footnotetext{
${ }^{1} \mathbf{x} \succeq \mathbf{y}$ means $x_{i} \geq y_{i}$ for $i=1, \ldots, p ; \mathbf{1}_{p}^{T} \equiv(1, \ldots, p)$.
}

most cases of practical interest $\mathbf{Q}$ is neither symmetric nor positivedefinite and, thus, the (3) is nonconvex. Therefore, there is no hope in finding systematically the global optima of (3). The SISAL algorithm, we introduce below, aims at "good" sub-optimal solutions.

Our first step is to simplify the set of constraint $\mathbf{1}_{p}^{T} \mathbf{Q Y}=\mathbf{1}_{n}^{T}$. We note that the vector $\mathbf{1}_{n}^{T}$ does not belong to the null space of $\mathbf{Y}$. Otherwise, the null vector would belong to the the affine hull of $\mathbf{M}$, what would imply that the columns of $\mathbf{M}$ would not be independent. Therefore, by multiplying the equality constraint on the right hand side by $\mathbf{Y}^{T}\left(\mathbf{Y} \mathbf{Y}^{T}\right)^{-1}$, we get $\left(\mathbf{1}_{p}^{T} \mathbf{Q Y}=\mathbf{1}_{n}^{T}\right) \Leftrightarrow\left(\mathbf{1}_{p}^{T} \mathbf{Q}=\mathbf{a}^{T}\right)$, where $\mathbf{a}^{T} \equiv \mathbf{1}_{n} \mathbf{Y}^{T}\left(\mathbf{Y} \mathbf{Y}^{T}\right)^{-1}$. The problem (3) simplifies, then, to

$$
\begin{aligned}
\mathbf{Q}^{*}= & \arg \min _{\mathbf{Q}}-\log |\operatorname{det}(\mathbf{Q})| \\
\text { s.t. : } & \mathbf{Q Y} \succeq \mathbf{0}, \quad \mathbf{1}_{p}^{T} \mathbf{Q}=\mathbf{a}^{T} .
\end{aligned}
$$

Instead of solving (4), we solve the following modified version:

$$
\begin{aligned}
\mathbf{Q}^{*}= & \arg \min _{\mathbf{Q}}-\log |\operatorname{det}(\mathbf{Q})|+\lambda\|\mathbf{Q Y}\|_{h} \\
\text { s.t. : } & \mathbf{1}_{p}^{T} \mathbf{Q}=\mathbf{a}^{T},
\end{aligned}
$$

where $\|\mathbf{X}\|_{h} \equiv \sum_{i j} h\left([\mathbf{X}]_{i j}\right)$ and $h(x) \equiv \max \{-x, 0\}$ is the socalled hinge function. Notice that $\|\mathbf{Q Y}\|_{h}$ penalizes the negative components of $\mathrm{QY}$ proportionally to their magnitude, thus playing the rule of a soft constraint or a regularizer. The amount of regularization is controlled by the regularization parameter $\lambda>0$. As already referred to, the soft constrained formulation yields solutions that are robust to outliers, noise, and poor initialization. Furthermore, by replacing $n \times p$ equality constraints by a regularizer, it opens the door to deal with large scale problems.

\section{SEQUENCE OF CONVEX SUBPROBLEMS}

Let $\mathbf{q} \equiv \operatorname{vec}(\mathbf{Q})$ denote the operator that stacks the columns of $\mathbf{Q}$ in the column vector $\mathbf{q}$. Given that vec $(\mathbf{A B})=\left(\mathbf{B}^{T} \otimes \mathbf{I}\right) \operatorname{vec}(\mathbf{A})=$ $(\mathbf{I} \otimes \mathbf{A}) \operatorname{vec}(\mathbf{B})$, where $\otimes$ denotes the kronecker operator, and defining $f(\mathbf{q})=-\log |\operatorname{det}(\mathbf{Q})|$, then (5) may be written as

$$
\begin{aligned}
\mathbf{q}^{*}= & \arg \min _{\mathbf{q}} f(\mathbf{q})+\lambda\|\mathbf{A q}\|_{h} \\
\text { s.t. : } & \mathbf{B q}=\mathbf{a},
\end{aligned}
$$

where $\mathbf{A}=\left(\mathbf{Y}^{T} \otimes \mathbf{I}\right)$ and $\mathbf{B}=\left(\mathbf{I} \otimes \mathbf{1}_{p}^{T}\right)$. The Hessian of $f$ is $\mathbf{H}=\mathbf{K}_{n}\left[\mathbf{Q}^{-T} \otimes \mathbf{Q}^{-1}\right]$, where $\mathbf{K}_{n}$ id the comutation matrix (i.e., $\mathbf{K}_{n} \operatorname{vec}(\mathbf{A})=\operatorname{vec}\left(\mathbf{A}^{T}\right)$ ). Since $\mathbf{H}$ has positive and negative eigenvalues, the above problem in nonconvex and thus hard.

Using a quadratic approximation for $f(\mathbf{q})$, we approximate (6) by computing a descent sequence $\mathbf{q}_{k}, k=0,1, \ldots$, with the following algorithm:

Algorithm 1 Sequence of strictly convex subproblems

1. Set $k=0$, choose $\mu>0$ and $\mathbf{q}_{0}=\operatorname{VCA}(\mathbf{Y})$.

2. repeat

3. $\quad l_{k}=f\left(\mathbf{q}_{k}\right)+\lambda\left\|\mathbf{A} \mathbf{q}_{k}\right\|_{h}$

4. $\quad \mathbf{g}=-\operatorname{vec}\left(\mathbf{Q}^{-1}\right)$

5. $\quad \mathbf{q}_{k+1} \in \arg \min _{\mathbf{q}} \mathbf{g}^{T} \mathbf{q}+\mu\left\|\mathbf{q}-\mathbf{q}_{k}\right\|^{2}+\lambda\|\mathbf{A q}\|_{h}$

6. $\quad$ s.t.: $\mathbf{B q}=\mathbf{a}$

7. if $f\left(\mathbf{q}_{k+1}\right)+\lambda\left\|\mathbf{A} \mathbf{q}_{k+1}\right\|_{h}>l_{k}$

8. $\quad$ find $\mathbf{q} \in\left\{\alpha \mathbf{q}_{k+1}+(1-\alpha) \mathbf{q}_{k}: 0<\alpha<1\right\}$

9. $\quad$ such that $f(\mathbf{q})+\lambda\|\mathbf{A q}\|_{h} \leq l_{k}$

10. $\quad \mathbf{q}_{k+1}=\mathbf{q}$

11. $k \leftarrow k+1$

12. until a stopping criterion is satisfied.

Algorithm 1 is initialized with the VCA [4] estimate. Line 4 computes the gradient of $f(\mathbf{q})$. Line 5 minimizes a strictly convex approximation to the initial objective function, where the term $f(\mathbf{q})$ was replaced by a quadratic approximation. The term $\left\|\mathbf{q}-\mathbf{q}_{k}\right\|^{2}$ ensures that $\left\|\mathbf{q}_{k+1}-\mathbf{q}_{k}\right\|^{2}$ does not grow unbounded. Lines 7 to 10 ensures that the objective function does not increase. To solve the minimization 5-6, we introduce in the next subsection a variable splitting augmented Lagrangian algorithm. 


\section{VARIABLE SPLITTING AND AUGMENTED LAGRANGIAN}

The optimization problem 5-6 of Algorithm 1 is equivalent to

$$
\begin{array}{ll}
\min _{\mathbf{q}, \mathbf{z}} & E(\mathbf{q}, \mathbf{z}) \\
\text { s.t. : } & \mathbf{B q}=\mathbf{a}, \quad \mathbf{A q}=\mathbf{z},
\end{array}
$$

where

$$
E(\mathbf{q}, \mathbf{z}) \equiv \mathbf{g}^{T} \mathbf{q}+\mu\left\|\mathbf{q}-\mathbf{q}_{k}\right\|^{2}+\lambda\|\mathbf{z}\|_{h} .
$$

In (7), the variable $\mathbf{q}$ was split into the pair $(\mathbf{q}, \mathbf{z})$ and linked through the constraint $\mathbf{A q}=\mathbf{z}$. The so-called augmented Lagrangian (AL) for this problem, with respect to the constraint $\mathbf{A q}=\mathbf{z}$ is given by

$$
\begin{aligned}
\mathcal{L}(\mathbf{q}, \mathbf{z}, \mathbf{d}, \tau) & \equiv E(\mathbf{q}, \mathbf{z})+\boldsymbol{\alpha}^{T}(\mathbf{A} \mathbf{q}-\mathbf{z})+\tau\|\mathbf{A q}-\mathbf{z}\|^{2}(8) \\
& =E(\mathbf{q}, \mathbf{z})+\tau\|\mathbf{A q}-\mathbf{z}-\mathbf{d}\|^{2}+c
\end{aligned}
$$

where $\boldsymbol{\alpha}$ is holds the Lagrange multipliers, $\mathbf{d}=-\boldsymbol{\alpha} /(2 \tau)$, and $c$ is an irrelevant constant. The AL algorithm consists in minimizing $\mathcal{L}$ with respect to $(\mathbf{q}, \mathbf{z})$ and then updating $\boldsymbol{\alpha}$, or, equivalently $\mathbf{d}$, as follows

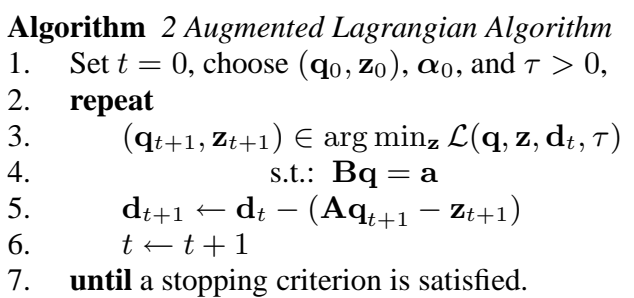

It has been shown that, with adequate initializations, the AL algorithm generates the same sequence as a proximal point algorithm (PPA) applied to the Lagrange dual of problem (7); for further details, see [20, 21] and references therein. Moreover, the sequence $\mathbf{d}_{k}$, for $k=0,1, \ldots$, converges to a solution of this dual problem and all cluster points of the sequence $\mathbf{z}_{k}$, for $k=0,1, \ldots$, are solutions of the (primal) problem (7) [20].

The exact solution of the optimization with respect to $(\mathbf{q}, \mathbf{z})$ in the line 3 of the Algorithm 2 is stil a complex task. However, the block minimizations with respect to $\mathbf{q}$ and with respect to $\mathbf{z}$ are very light to compute. Based on this, we propose the following modification of Algorithm 2:

\section{Algorithm 3 Alternating Split AL}

1. Set $t=0$, choose $\left(\mathbf{q}_{0}, \mathbf{z}_{0}\right), \boldsymbol{\alpha}_{0}$, and $\tau>0$,

2. repeat

3. $\quad \mathbf{q}_{t+1} \in \arg \min _{\mathbf{q}} \mathbf{g}^{T} \mathbf{q}+\frac{\mu}{2}\left\|\mathbf{q}-\mathbf{q}_{k}\right\|^{2}+\frac{\tau}{2}\left\|\mathbf{A q}-\mathbf{z}_{t}-\mathbf{d}_{t}\right\|^{2}$

$$
\text { 4. s.t.: } \mathbf{B q}=\mathbf{a}
$$$$
\text { 5. } \quad \mathbf{z}_{t+1} \in \arg \min _{\mathbf{z}} \frac{1}{2}\left\|\mathbf{A} \mathbf{q}_{t+1}-\mathbf{z}-\mathbf{d}_{t}\right\|^{2}+\frac{\lambda}{\tau}\|\mathbf{z}\|_{h}
$$$$
\text { 6. } \quad \mathbf{d}_{t+1} \leftarrow \mathbf{d}_{t}-\left(\mathbf{A} \mathbf{q}_{t+1}-\mathbf{z}_{t+1}\right)
$$$$
\text { 7. } \quad t \leftarrow t+1
$$

8. until stopping criterion is satisfied.

The solution of the quadratic problem with linear constraints 3-4 is

$$
\mathbf{q}_{t+1}=\mathbf{F}^{-1} \mathbf{b}-\mathbf{F}^{-1} \mathbf{B}^{T}\left(\mathbf{B} \mathbf{F}^{-1} \mathbf{B}^{T}\right)^{-1}\left(\mathbf{B} \mathbf{F}^{-1} \mathbf{b}-\mathbf{a}\right),
$$

where

$$
\begin{aligned}
& \mathbf{F} \equiv\left(\mu \mathbf{I}+\tau \mathbf{A}^{T} \mathbf{A}\right) \\
& \mathbf{b} \equiv \mu \mathbf{q}_{t}-\mathbf{g}+\tau \mathbf{A}^{T}\left(\mathbf{z}_{t}+\mathbf{d}_{t}\right)
\end{aligned}
$$

The minimization with respect to $\mathbf{z}$, in line 5 , is, by definition the proximity operator of of the convex function $\|\mathbf{z}\|_{h}$ [22], which is similar to the soft threshold function but applied just to the negative part of its argument:

$$
\mathbf{z}_{t+1}=\text { soft }_{-}\left(\mathbf{A q}_{t+1}-\mathbf{d}_{t}, \mu / \tau\right)
$$

where $\operatorname{soft}_{-}(x, \beta)=(\max \{|x+\beta / 2|-\beta / 2,0\})(x /|x|)$ is a thresholding/shrinkage function and, for a matrix $\mathbf{X}$, soft_( $\mathbf{X}, \beta)$ is the componentwise application of $\operatorname{soft}_{-}(\cdot, \beta)$. We note that computations $(10)$ and $(12)$ are very light: In the first case, all matrices involved are $p^{2} \times p^{2}$ and are not iteration dependent. In the second case, the function soft- $(\cdot)$ takes negligible time.

The iterations of Algorithm 3 are much faster than that of Algorithm 2. There is, however, the question of convergence. The answer to this question turns out to be positive, a result that can be proved via the equivalence between the alternating split $A L$ algorithm just described and the so-called Douglas-Rachford splitting method, applied to the dual of problem (7); see [23] Theorem 8], [21].

In conclusion, the pseudo-code for SISAL is given by Algorithm 1 with the step 5 replaced by Algorithm 3.

\section{EXPERIMENTAL RESULTS}

This section presents results obtained with SISAL, MVSA (hard version) [15], MVES [14], and VCA [4] applied to simulated data sets. The SISAL regularization parameters was set to $\lambda=10$, The remaining parameters were set to $\tau=1$ and $\mu=10^{-4}$. Although these values may be far from optimal, they led to excellent results. The data was generated according to the linear observation model (1). The abundance fractions are Dirichlet distributed with parameter $\mu_{i}=1$, for $i=1, \ldots, p$. The mixing matrix $\mathbf{M}$ is randomly generated with i.i.d. uniformly distributed elements. To ensure that no pure pixel is present, we discarded all pixels with any abundance fractions larger than 0.8 . The signal-to-noise ratio (SNR) defined as $\|\mathbf{Y}\|_{F}^{2} /\|\mathbf{N}\|_{F}^{2}$, where $\|\cdot\|_{F}^{2}$ denotes the Frobenius norm and $\mathbf{N}$ is zero-mean Gaussian additive noise, was set to $\mathrm{SNR}=40 \mathrm{~dB}$.

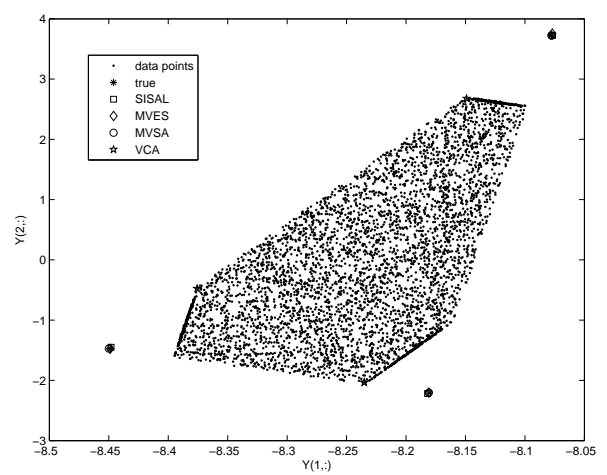

(a)

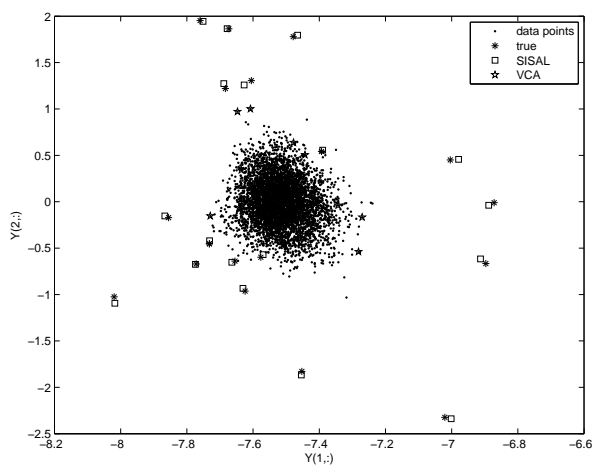

(b)

Fig. 2. Unmixing results for (a) $p=3$ and (b) $p=20$ number of endmembers for SISAL, MVSA, and MVES algorithms. Dots represent spectral vectors; all other symbols represent inferred endmembers by the unmixing algorithms. The unmixing problem with $n=10000$ spectral vectors and $p=20$ endmembers is far beyond the reach of MVSA, and MVES. 
Table 1. Comparison of SISAL, MVSA, and MVES algorithms for different number of endmembers and sample size $n=10000$. The time is in seconds, the symbol "*" means the algorithm ran out of memory, while $\dagger$ indicates that the algorithm was aborted before converging. Note the $O(n p)$ time complexity of SISAL.

\begin{tabular}{c||c|c||c|c|c|c}
\hline \multirow{2}{*}{$\mathrm{p}$} & \multicolumn{2}{|c|}{ SISAL } & \multicolumn{2}{c|}{ MVSA } & \multicolumn{2}{c}{ MVES } \\
\cline { 2 - 7 } & $\|\varepsilon\|_{F}$ & $\mathrm{~T}$ & $\|\varepsilon\|_{F}$ & $\mathrm{~T}$ & $\|\varepsilon\|_{F}$ & $\mathrm{~T}$ \\
\hline 3 & 0.03 & 3 & 0.02 & 2 & 0.03 & 2 \\
6 & 0.08 & 4 & 0.10 & 4 & 0.10 & 56 \\
8 & 0.07 & 10 & 0.18 & 10 & 0.24 & 296 \\
10 & 0.13 & 10 & 0.25 & 24 & $\dagger$ & $>1500$ \\
12 & 0.15 & 14 & $*$ & $*$ & $\dagger$ & $\gg 1500$ \\
20 & 0.18 & 16 & $*$ & $*$ & $\dagger$ & $\gg 1500$ \\
\hline
\end{tabular}

Fig. 2 shows a projection on a subspace of the true endmembers, of the SISAL, MVSA, and MVES estimates and the spectral vectors. The data set has size $n=10000$ and a number of endmembers $p=3$, (part a), and $p=20$, (part b). In part $\mathrm{b}$, we just plot SISAL results because MVES takes hours for $p \geq 10$, and MVSA exhausts the memory for $p \geq 12$.

Notice the high quality of SISAL, MVSA, and MVES estimates in both scenarios. This is not the case with VCA, as it was not conceived for non-pure pixel scenarios. VCA plays, however, a valuable rule in the SISAL, MVSA, and MVES initializations.

Table 1 shows the times in seconds and the Frobenius norm $\|\varepsilon\|_{F}$ of the endmember error matrices $\widehat{\mathbf{M}}-\mathbf{M}$. The experiments were performed on an PC equipped with a Intel Core Duo $3 \mathrm{GHz}$ CPU and 4 GB of RAM. The errors are comparable. However, MVES takes much longer and we could not run it for more than $p=10$. MVSA ran out or memory for $p>10$. The time SISAL takes is well approximated by a $O(n p)$ bound, what could be inferred from its structure.

\section{CONCLUSIONS}

SISAL, a new algorithm for hyperspectral unmixing method of minimum volume class, was introduced. The unmixing is achieved by finding the minimum volume simplex containing the hyperspectral data. This optimization problem was solved by a sequence of variable splitting augmented Lagrangian optimizations. The algorithm complexity is $O(n p)$, where $n$ is the number of spectral vectors and $p$ is the number of endmembers what is much faster than the previous state-of-the-art, allowing to solve problems far beyond the reach of SISAL's competitors.

\section{ACKNOWLEDGMENT}

The author thanks T.-H. Chan, C.-Y. Chi, and W.-K. Ma, for providing the code for the MVES algorithm [14].

\section{REFERENCES}

[1] D.G. Manolakis N. Keshava, J.P. kerekes and G.A. Shaw, "Algorithm taxonomy for hyperspectral unmixing," Proc. SPIE Vol.4049, Algorithms for Multispectral, Hyperspectral, and Ultraspectral Imagery, vol. VI, pp. 42, 2000.

[2] J. Nascimento and J. Bioucas-Dias, "Vertex component analysis: A fast algorithm to unmix hyperspectral data," IEEE Transactions on Geoscience and Remote Sensing, vol. 43, pp. 898-910, 2005.

[3] R.M. Perez A. Plaza, P. Martinez and J. Plaza, "A quantitative and comparative analysis of endmembr extraction algorithms from hyperspectral data," IEEE Transactions on Geoscience and Remote Sensing, vol. 42, pp. 650-663, 2004.

[4] J. Nascimento and J. Bioucas-Dias, "Does independent component analysis play a role in unmixing hyperspectral data?," IEEE Transactions on Geoscience and Remote Sensing, vol. 43, pp. 175-187, 2005.
[5] L. Miao and H. Qi, "Endmember extraction from highly mixed data using minimum volume constrained nonegative matrix factorization," IEEE Transactions on Geoscience and Remote Sensing, vol. 45, pp. 765-777, 2007.

[6] M. Craig, "Minimum-volume transforms for remotely sensed data," IEEE Transactions on Geoscience and Remote Sensing, vol. 32, pp. 542-552, 1994.

[7] J. Nascimento and J. Bioucas-Dias, "Hyerspectral unmixing algorithm via dependent component analysis," IEEE Internationla Geoscience and Remote sensing Symposium, pp. 4033-4036, 2007.

[8] N. Dobigeon, J.-Y. Tourneret, and C.-I Chang, "Semi-supervised linear spectral unmixing using a hierarchical Bayesian model for hyperspectral imagery," IEEE Transactions on Signal Processing, vol. 56, no. 1, pp. 2684-2695, 2008.

[9] S. Moussaoui, H. Hauksdóttir, F. Schmidt, C. Jutten, J. Chanussot, D. Brie, S. Douté, and J. A. Benediksson, "On the decomposition of Mars hyperspectral data by ICA and Bayesian positive source separation," Neurocomputing, 2008, accepted.

[10] M. E. Winter, "N-FINDR: an algorithm for fast autonomous spectral endmember determination in hyperspectral data," in Proc. of the SPIE conference on Imaging Spectrometry V, vol. 3753, pp. 266-275, 1999.

[11] J. Boardman, "Automating spectral unmixing of AVIRIS data using convex geometry concepts," in JPL Pub.93-26,AVIRIS Workshop, vol. 1, pp. 11-14, 1993.

[12] R. Perez A. Plaza, P. Martinez and J. Plaza, "Spatial/spectral endmember extraction by multidimensional morphological operations," IEEE Transactions on Geoscience and Remote Sensing, vol. 40, pp. 20252041, 2002

[13] C.-I. Chang, C.-C. Wu, W. Liu, and Y.-C. Ouyang, "A new growing method for simplex-based endmember extraction algorithm," IEEE Transactions on Geoscience and Remote Sensing, vol. 44, no. 10, pp. 2804-2819, 2006.

[14] C.-Y. Chi T.-H. Chan and W.-K. Ma, "A convex analysis based minimum-volume enclosing simplex algorithm for hyperspectral unmixing," in IEEE International Conference in Acoustics, Speech and Signal Porcessing-ICASSP'2009, Taiwan, 2009.

[15] J. Li and J. Bioucas-Dias, "Minimum volume simplex analysis: a fast algorithm to unmix hyperspectral data," in IEEE International Geoscience and Remote sensing Symposium -IGARSS2008, Boston, 2008.

[16] Liming Zhang Xutao Tao, Bin Wang and Jian Qiu Zhang, "A new scheme for decomposition of mixed pixels based on nonnegative matrix factorization," IEEE Internationla Geoscience and Remote sensing Symposium, pp. 1759-1762, 2007.

[17] M. Figueiredo, J. Bioucas-Dias, and M. Afonso, "Fast image restotarion using variable splitting and constrained optimization," Technical report, Instituto Superior Técnico, TULisbon, Lisbon, 2009.

[18] T. Goldstein and S. Osher, "The split Bregman method for 11 regularized problems," Technical Report 08-29, Computational and Applied Math., Univ. of California, Los Angeles, 2008.

[19] J. Bioucas-Dias and J. Nascimento, "Hyperspectral subspace identification," IEEE Transactions on Geoscience and Remote Sensing, vol. 46, no. 8,2008

[20] A. Iusem, "Augmented Lagrangian methods and proximal point methods for convex optimization," Investigación Operativa, vol. 8, pp. 1149, 1999.

[21] S. Setzer, "Split Bregman algorithm, Douglas-Rachford splitting, and frame shrinkage," in Proceedings of the Second International Conference on Scale Space Methods and Variational Methods in Computer Vision, 2009, LNCS Springer.

[22] P. Combettes and V. Wajs, "Signal recovery by proximal forwardbackward splitting," SIAM Journal on Multiscale Modeling \& Simulationg, vol. 4, pp. 1168-1200, 2005.

[23] J. Eckstein and D. Bertsekas, "On the DouglasRachford splitting method and the proximal point algorithm for maximal monotone operators," Mathematical Programming, vol. 5, pp. 293-318, 1992. 